\title{
MEMS Technology \\ MEMS Development in a Past Decade and its Future Prospects
}

Keywords

Microelectromechanical Systems (MEMS), Microactuator,

Hiroyuki Fujita

Micromachining, Microsensors, Application

The University of Tokyo

Summary

\begin{abstract}
A history of microelectromechanical system(MEMS) research and development from 1987 is reviewed Major achievements in MEMS research are listed in the time sequence. The achievements include microactuators, microsensors, micro miniature motion-systems (e.g. microrobots), applications and the fabrication technology involved Future prospects of MEMS in contributing the 21th Century society are expected in three areas such as (1) wider distribution of and casier acoess to information, (2) making human activities more compatible with environment and (3) improvement in social welfare. The technological issues for future development of MEMS are also discussed.
\end{abstract}

\section{INTRODUCTION}

One of the dreams of mankind is micro miniature machines which are almost invisible and with which we can exlore the microcosomos. Such machines, so called mictoelectromechanical systems(MEMS) in United Sates and micromachines in Japan, are composed of both mechanical devices and electrical devices. While the miniaturization of electrical devices such as sensors and electronic circuits was well established technically, the study of mechanical devices such as micro mechanisms and microactuators began unly a decade ago when the IEEE workshop on micro electro mechanical systems (MEMS) was founded Since then, researchers have achieved remarkable progress. The successful fabrication and operation of mictoactuators and micro mechanical parts confimed the feasibility of the concept. The development of IC-compatible micromachining technology enabled us to produce MEMS[1-3]. Although the small size of mechanical components of the system is a very distinctive feature of this emerging technology, it has other, maybe even more attracive, features. The three characteristic features or the three "M"s of the technology are[2]:

\section{Miniaturization}

Multiplicity

Microelectronics

Miniaturization is clearly essential. However, the mere miniaturization of macroscopic machines is not the most advantageous way to realize MEMS because of the scaling effect. In the microworld, the same physical laws as in the macroworld govem the machine behavior but the relative importance of different effects changes dramatically when the size of machines decreases. The way the machine works in the microworld may also different. Like a swarm of ants carrying a large food, cooperative work of many micro elements can perform a large task, even when one single device can only produce small force or perform simple motion. Multiplicity is the key to successful microsystems. The integration of microelectronics is essential for micro moving elements to cooperate with each other and to perform a given task.

In order to realize MEMS with above mentioned features, fundamental technological issues are materials, machining processes and mictomechanical devices. There are two kinds of machining processes; one is based on semiconductor technologies and the other on mechanical means. Devices include sensors, actuators and integrated circuits. Detailed research items in these issues are shown in Fig. 1. Please note that these issues are not investigated separately but in close relations between each other.

Using these technologies in Fig. 1, we may utilize MEMS in some applications. Figure 2 shows the prospective applications in optics, transportation and aerospace, robotics, chemical analysis systems, biotechnologies, medical engineering, microscopy using scanned mirco
probes. Most of the applications have a common feature in that only very Jight objects such as mirrors, heads, valves, cells and microprobes are manipulated and that little physical interaction with the external environment is necessary. One reason is that present microactuators are still primitive and large forces cannot be transmitted to the extemal world. The other reason is difficulty in packaging.

The micromachining technology has quite a long history. Table 1[3] summirizes major inventions before 1987 when the first MEMS workshop was held. The achievements after 1987 are listed in Table 2 together with the names of places where MEMS Workshops and Transducers (International Conference on Slidstate Sensors and Actuators) Conferences were held. Papers presented at MEMS Workshops are included as much as possible while those at Transtucer Conferences are very limited because of the space. Also, reference to each paper is not able to be addressed. Papers are categolized in accordance with the items shown in Figs. 1 and 2.

Table 1 History of micromachining (before 1986) 


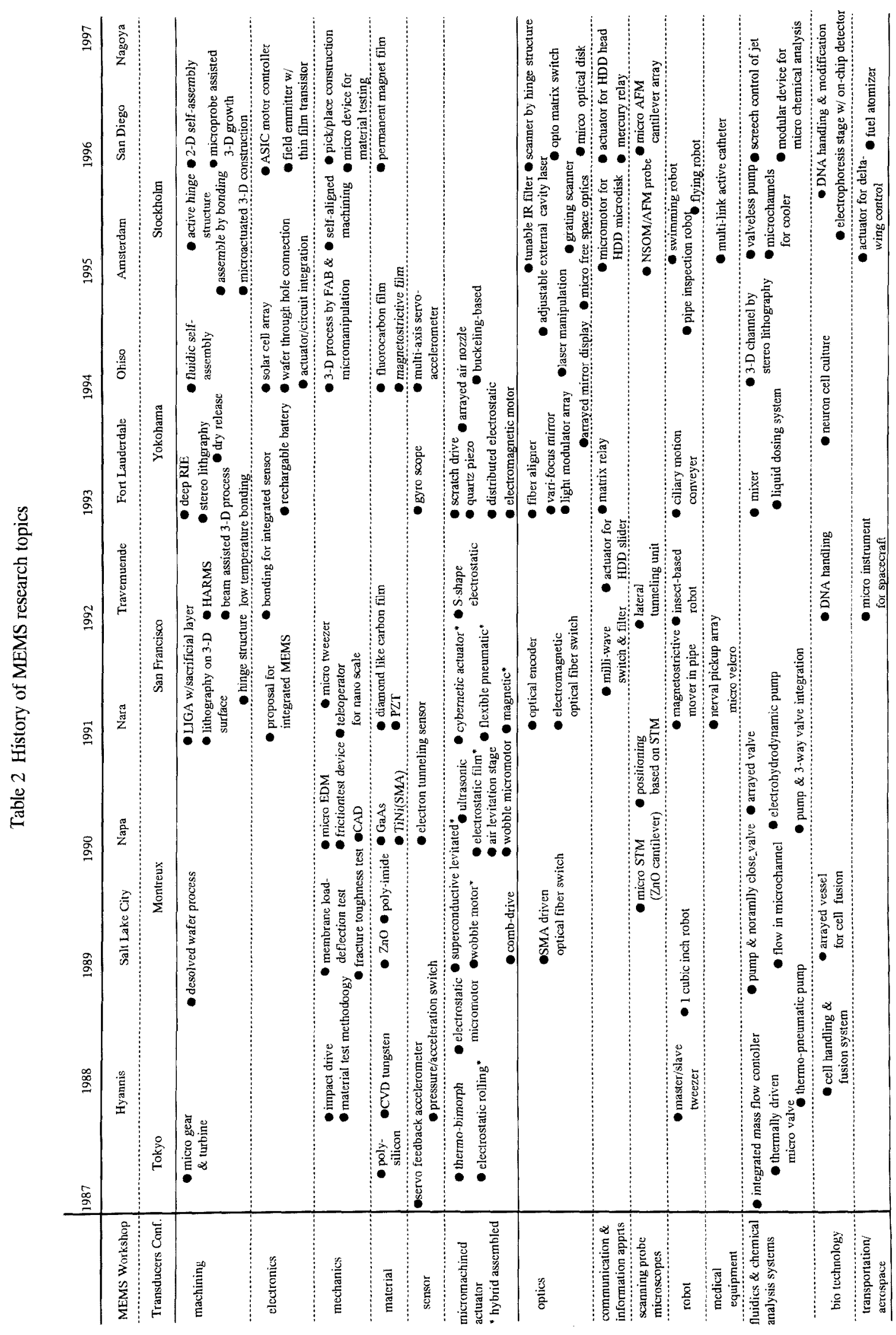




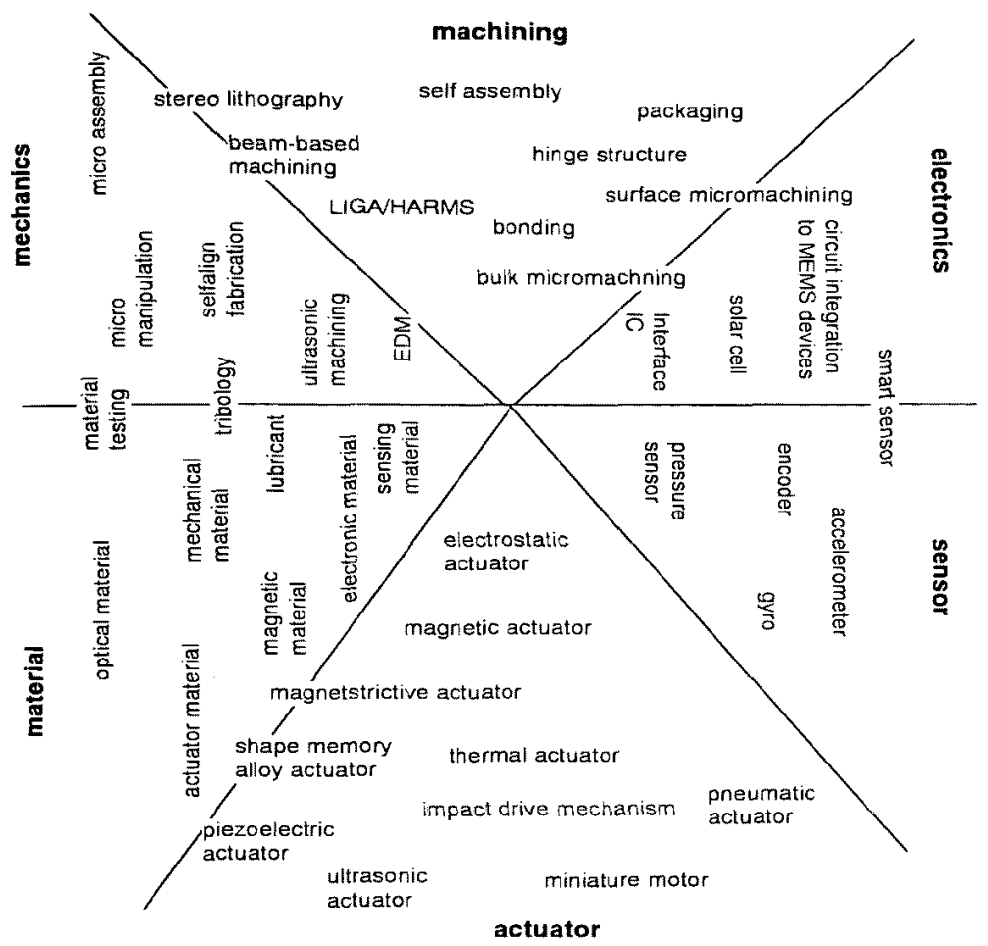

Fig. 1 A map of MEMS devices and technologies

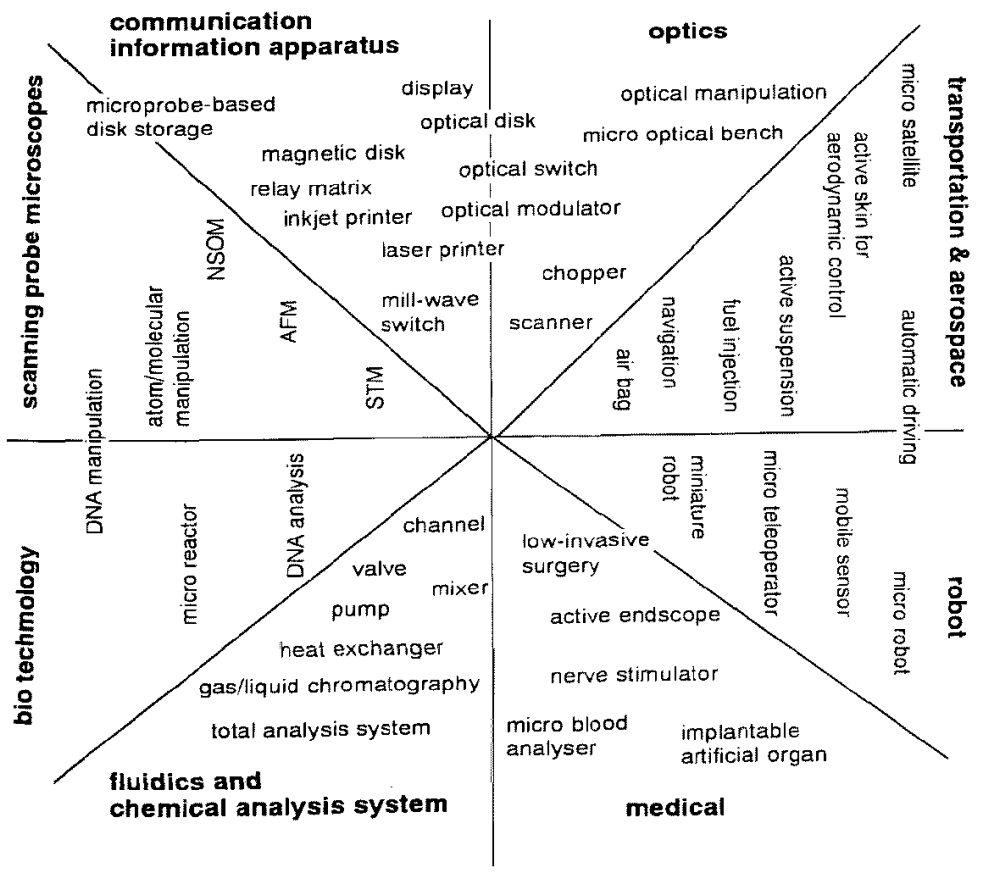

Fig. 2 A map of MEMS applications

\section{DEVICES AND TECHNOLOGIES}

\subsection{Materials for MEMS}

Silicon is the most commonly used material in micromachining [4] because (1) the process is well established, (2) it has good mechanical properties, and (3) integration with electronic and sensors is possible. The bulk micromachining technology is used for single crystal silicon and the surface micromachining mainly for poly-silicon flims but some times for single crystal silicon films on SOr wafers. Other materials have also been used in micromachining for specific purposes such as actuation, light emission, and lubrication. Table 3 summarizes such materials and their useful characteristics. Researchers have developed batch fabrication processes for all the materials in the table, so they reiterate the abovementioned features of micromachining.

\subsection{Micromachining}

Microstructures fabricated by surface micromachining are planar in nature and have thickness of up to $10 \mu \mathrm{m}$ in most cases. Some applications require thicker structures or three-dimensional-complicated structures. LIGA process utilizes deep X-ray lithograpy, electroplating and molding to make thick structures[3]. UV-lithography with special resisit, photosensitive polyimide and deep reactive ion etching (RE) have also used to make high aspect ratio (the ratio between height and width of a structure) microstructures and micromold. If plated metals are used to make replicas, resulted structures are called HARMS, meaning high aspect ratio metalic structures. Poly-silioon by LPCVD (low pressure chemical vapor depsition) can be used to have replicas of deep RIE'ed structures on silicon wafers. Wafer bonding technologies combined with deep RIE have developed to build 3-D structures.

Modifications of surface micromachining have been attempted, too. One techniquc is to fold up micromachined plates from the substrate to construct a 3-D structure. The plate is released from the substrate and reconnected by hinges [5], flexible films or even active hinges made by conductive polymers. Such structures as a coner cube reflector and a micro "ant-based robot" were fabricated Surface-Inictomachined polysilicon films were deformd permanetly by bending them elastically and, then, heating them by letting current run through them. In other trials, overhanging structures were made. Microscopic tweezers made of polysilicon protrude $400 \mu \mathrm{m}$ from the edge of a wafer[6] A single-celled protozoa, a euglena, was held by this microgripper.

Electron beams, laser beams or ion/atomic beams can assist selective growth/solidification/etching of materials. Three-dimensional structures in arbitrary shapes can be fabricated[7]. Lithography on 3-D surfaces is possible by using these beams. Micostructures in nanometer sizes were made with the electron bean from the tip of a scanning tunneling microscope (STM). Mechanical processes such as electro discharge machining(EDM), ultrasonic machining and guliding for micro structures have also 
Table 3 Materials in Micromachining

material usage process characteristics
$\mathrm{Ni}, \mathrm{Cu}, \mathrm{Au}$ structure (electro) plating thick structures
tungsten structure thin film not attacked by HF
polyimide structure thin film soft \&flexible, optical guide
quartz actuation anisotropic piezoelectricity, insulator
$\mathrm{ZnO}$ actuation thin film piezoelectricity
$\mathrm{PZT}$ actuation thick film large piezoelectricity
$\mathrm{TiNi}$ actuation thin film shape memory allay
TbFe, SmFe actuation thin film magnetostriction
$\mathrm{CoNiMn}$ actuation thin film permanent magnet
GaAs optics thin film LASER, LED, detector
DLC lubrication thin film low friction \& wear
fluorocarbon lubrication thin film reduce friction \& wear

demonstrated Sometimes such machining processes require parts handling; for this micro manipulation is studied. Unfortunately there is a trade-off between batch fabrication capability and 3-D complicated machining capability.

\subsection{Microactuators}

A microactutor is the key device for MEMS to perfrom physical functions. Because of the scaling consideration[8], the electromagnetic force which is most commonly used in the macro actuators is not the only driving force for microactuators but also many microactuators utilizes other driving principles such as the electrostatic force, the piezoelectric force, the magnetostrictive force, the shape memory effect and themal expansion.

Each actuation princple has its own advantages and disadvantages. The choice and the optimization should be made according to the requirements of applications. Generally speaking, the electrostatic actuator is more suitable to perform tasks which can be completed within a chip (positioning of devices/heads/probes, sensors with servo feedback, light deflection and modulation, etc), since it is easily integrated on a chip, easily controlled and consumes little power. On the contrary, the other types of actuators are more robust, prochce large force and are suitable to perform external tasks (propulsion, manipulation of objects, etc.)

\subsection{Electrostatic micromotors/actuators}

The first electrostatic mictomotors with diameters of $60-120 \mu \mathrm{m}$ were developed by L.-S. Fan, Y.-C.Tai, and R.S. Muller [9]. It is called a side-drive type motor, since it utilizes the electrostatic force which acts between the edges of the rotor and the stator both made with polysilicon. Rotational speed was on the order of $500 \mathrm{rpm}$. The speed is relatively low bacause of the large friction between the rotor and the shaft, although a silicon nitride film was deposited on the sliding surface to reduce the friction. Later impovement by Mehregany, et al. [8] enabled the rotational speeds up to $15,000 \mathrm{rpm}$ and continuous operation for more than a week. They reduced the clearance between the rotor and the shaft, formed three dimples under the rotor for both support and electrical contact.

Even for the improved micromotors, friction was a major problem. One solution is to replace the sliding contact at the center with rolling contact. A type of the micromotor, so called a wabble motor[10], was designed on this solution and realized by surface micromachining. Its rotor is a smooth ring and, by electrostatic attraction, rotates eccentrically without slipping at the contact with shaft. Since the circumferential distance of the rotor hole is slightly longer than that of the shaft, the rotor really revolves a fraction of a circle after one eccentric rotation. This results in two advantages of the motor, e.g. reduction of friction and higher torque at low speed. The usage of rolling motion in many other geometories was demonstrated, although fabrication processes for these actuators were not IC based nor fully IC-compatible.

Another way to avoid the effects of friction is with elastic supports. A comb-drive actuator is the most widely known[11]. Its moving part is supported by double-fold beams and actuated linearly by interdigiting comb-like structures. The electrostatic force to increase the overlapping is generated when voltage is applied between the suspended and fixed combs. Typical displacement is $10 \mu \mathrm{m}$ and the generated force is $10 \mu \mathrm{N}$.

\subsection{Other Driving Principles}

Microactuators which utilize such driving principles as the piezoelectric force, the shape memory effect, thermal expansion(gas[12], liquid, solid), liquid to gas phase transition and the electomagnetic force have been developed. In terms of reducing friction, most of them moves elastically with somc exceptions. The ultrasonic micromotor/actuator utilizes the standing wave to drive the rotor/slider. Coordinated vibrations of cantilevers can carry objects through mechanical oontact[13]. Levitation by repulsive force between the permanent-magnet and the superconducting material[14], by air pressure from small holes and the controlled electrostatic force were demonstrated. Controlled air flow from micronozzles could levitate and carry a flat object[15]. Recoil of a small mass which is moved quickly by a piezoactuator can drive a main body in a stepwise manner[16]. The position of the main body is kept still by static friction when the small mass returns to its initial position slowly.

\subsection{Arrayed actuators}

If we want to have MEMS to perform a macroscopic task, the key idea is to coordinate simple motions of many microactuators. Even when each moving step is small, accumulation of many steps covers large distance. A heavy load may be distributed among many actuators which produce only small force. Flexibility of motion, expandability and immunity against failure of elements can be achieved One of the major problems in present microactuators, the problem of friction can also be solved. Friction in micro scale prohibits us from using gears and joints because they waste too much energy. Suspended actuators do not suffer from friction but have limited motion range up to a few tens of micrometers. If many such microactuators are ananged in series and parallel, the overall structure can produce larger force and displacement and perform more complicated functions than each simple actuator. Because these actuators are driven directly, energy loss associated with transmission of motion is minimal. They can even utilize the friction between an object and them to transmit driving force. Arrays of micro valves, a type of electrostatic actuators with many small force generating elements, arrays of cantilever actuators which vibrate in synchronization and convey objects $[13]$ and a in-plane conveyance system using controlled air flow from arrayed nozles on the substrate[15] were operated successfully. A projection display based on arrayed movable micromirrors is commercialized[17]. Miniaturized electromagnetic relays are arrayed for telephone switching.

\section{APPLICATIONS}

\subsection{Optics}

Petersen, et al.[4] demonstrated deflecting light beams by small cantilevers driven by electrostatic force in 1977 . Since then, optical-fibe switches, its aligner and an adjustable miniature Fabry-Perot interferometer which acted as a tunable filter, an extemal cavity for laser diode and a passive modulator were reported. Integrated optic technology was used to fabricate a one-chip optical microencoder[18]. A display based on defraction gratings was developed Three-dimensional hinged stnuctures were used to build free space optical systems on a silicon wafer[19].

\subsection{Fluidics}

This is another application with long research history. An integrated mass flow controller[20] was developed more than ten years ago. Integrated micro dosing systems were built. Chemical analysis systems are under intensive study. The control of macto aerodynamics[2], e.g. formation of voltex, by microactuators on the surface or around a nozzle has demonstrated. The positive growth of the disturbance given by the small motion of a microactuator enables effective control of macroscopic phenomena.

\subsection{Communication and Information Apparatus}

Many optical MEMS devices have been developed for communication systems, especially for fiber-communication networks[21]. MEMS displays based on movable mirrors[17] and micromachined filed emmiter 
arrays were developed. One of the earliest and the most successful application of MEMS is the themal ink jet printers. In hard disk data storages, sliders, suspensions and actuators for fine tracking have been fabricated by micromachining technologies[22]. Micro optical disk was also tried.

\subsection{Biotechnology}

The typical dimensions of biological objects are around $1-10 \mu \mathrm{m}$ for biological cells and nano meters in thickness by microns in length for macro molecules. The electric field distribution obtained by microfabricated electrodes can be controlled in the same order of the object size and is suitable for manipulating them. Washizu, et al.[23] developed a cell fusion system using both a micro fluidic system and manipulation with the electric field. They also succeeded in orienting DNA molecules along the field and modify them by enzyme attached to a laser manipulated bead. Arrays of small vessels for cell fusion and culture were fabricated. MEMS for DNA mutiplication and detection are under development. Microdroplets can be delivered by a pump based on ink jet printing heads.

\subsection{Scanning prabe microscopes}

A micromachined STM (scanning tunneling microscope) composed of a piezoelectrically driven cantilever was successfully operated to take the atomic image of a graphite surface[23]. Another type of the micro STM utilized electrostatic actuators. Micromachined devices which contol tunneling current have been applied to detectors for highly sensitive sensors[25]. Arrays of AFM (atomic force microscope) cantilevers and STM cantilevers were fabricated and under test. As was mentioned above, STM probes are also useful for lithography in $\mathrm{nm}$ scale and growth of thin and sharp micro structures. Even an atom can be manipulated by SPM (scanning probe microscopes). Analysis of physical/chemical interactions based on observation of a single atom/molecule and fabrication of new compound materials by co-called "atom-craft" will be realized by micromachined SPM's.

\section{ISSUES FOR FUTURE DEVELOPMENT OF MEMS}

\subsection{Application-pull}

Major issues of society in the 21 st century will be:

(1) wider distribution of and easier access to information,

(2) making human activities more compatible with environment and (3) improvement in social welfare.

MEMS, with above mentioned features, are expected to provide technological breakthroughs for these issues. Bearkthroughs would be in five folds: machine intelligence, downsizing and parallelism, biomimetics, informatics and environment monitoring / preservation.

It is necessary, however, to find profitable commercial applications in a short time in order to accelerate research and development of MEMS. This is particulary essential for industries. MEMS research is supported mainly by govermental projects in US, Europe and Japan now. For the future development of the technology, investments in private sectors are indispensable. First generation products and matured prototypes are being introduced in the following areas:

(1) Information apparatus such as displays, printers and data storage devices,

(2) micro-optical devices for global communication networks, and (3) micro liquid handling systems for medical analysis and environmental monitoring.

We have to increase the number of products in other areas while pushing above mentioned areas. For instances:

4.2 MEMS for smart homes: A future room may be equipped with MEMS to maintain aminity. Illumination, temperature, humidity, with MEMS to maintain aminity. In MEMS for this purpose defects conditions of the room using its temparature sensor, humidity sensor, air flow sensor, infrared sensor and microphone. Electronic cjrcuits in the system determine appropriate response based on sensor signals and infromation from neighboring
MEMS. The effectors of the system such as a heater, a ventilator, a lamp and a speaker, which are all miniaturized and arrayed, can adjust the condition to achieve maximum aminity. Misrosystems can also be utilized for security and safety surveillance. The total system will probably have adaptive capability to trim its performance in accordance with the owner's preference and habit

4.3 MEMS for transportation and aerospace: Automibile has been and will be one of the major application field of MEMS. MEMS will be used not only to make cars smarter but also to realize intelligent transportation systems. Aircraft design may be drastically changed by the introduction of MEMS[2]. The initial size of vortices of air is in the order of a few tens of micrometers. Therefore, if arrays of MEMS each of which can detect and extinct the vortex are embedded on the surface of a aircraft, it is possible to reduce aerodynamic drag dramatically. The arrays can also generate drag intentionally to control the posture of the aircraft. Size and weight are the most important limitation for spacecrafts. Mycrosystems can be utilized to reduce the size and weight of apparatus with mainlaining the same functionality as conventional systems. The concept of microsatellites has been discussed recently[26] One day, a MEMS equipped with micro sensors for space observation, processors, anntenna, micro rockets and controllers, etc may be fabricated on a substrate and launched, making a "flying wafer".

\subsection{Technology-push}

Devices such as micromotors and microactuators have been proven. It is necessary to demonstrate real MEMS composed of mechanical and electrical elements. For instance, a micromotor is useful only when it is connected to elements to drive and controled precisely at desired speed The design method and integrate fabrication technology of MEMS must be established.

A new area of micro science and engineering(MSE) should be establish in order to provide the theoretical foundations for the development of micromachines. MSE is the extension of conventional science and technology toward the microscopic world. The MEMS area can benefit from the knowledge offered by a broad range of scientific and engineering disciplines, such as organic and inorganic material science, process engineering, device fabrication, system design and control, and variety of application fields.

The scaling effect is one of the fundamental issues in building micromachines. The frictional force dominates the inertial force in micro scale and prevent micto gears or rotors from moving smoothly, if moving at all. Friction and tribology in micro domain must be well understood. There are more issues such as accumulation of data of material properties in micro scales, standard evaluation methods for MEMS, a convenien1 performance index for MEMS which is similar to "instructions per seoond" for computers and "design rules" for IC's, and advanced new materials for actuators, structures and sensors.

\subsection{System archtechture for MEMS}

The integration of sensors, actuators and controllers lead to the concept of autonomous distributed micromachines(ADM) as an system architecture suitable for MEMS[27]. An autonomous distributed system is a system which is composed of many smart subsystems called individuals. An individual can gather information with its sensors and through communication from neighboring individuals and sometimes from the overall system. It independently determines its behavior based on the information. The way they decide their behaviors is to cooperate each other in order to complete the objectives of the overall system. The ADM are composed of many smart modules working as individuals which are clever enough to control their own actuators and to oooperate with each other. Design, low cost fabrication and control issues of ADM should be studied. Following the example of Giant Microelectronics such as liquid cristal displays, we will be able to build a Giant MEMS, a macroscopic system composed of many smart microsystems realized by MEMS technologies.

\section{CONCLUSION}

MEMS will have profound impact in the future society. It is necessary to continue and enhance research activities in both fundamental and application-oriented areas. Fusion of knowledge in different disciplines 
is essential for well-balanced and accelerated growth of the technology. I strongly believe that the interational collaboration in science and technology of MEMS together with healthy competition in their commercialization field will lead to gigantic success of MEMS. Part of the content is owed to discussion with Dr. K. J. Gabriel at DARPA.

\section{REFERENCES}

(Manuscript received March 31, 1997)

[1] R.T. Howe, R.S. Muller, K.J. Gabriel, W.S.N. Trimmer, 'Silicon Micromechanics: Sensors and Actuators on a Chip', IEEE Spectrum, June 1991, pp. 29-35.

[2] K.J. Gabriel, "Engineering Microsoopic Machines", Scientific American, vol. 260, no.9, pp. 118-121 (1995.9).

[3] M. Esashi, H. Fujita, I. Igarashi, S. Sugiyama, "Micromachining and Micromechatronics" Baifu-kan Publ, Tokyo (1994) in Japanese.

[4] K. Petersen, "Silicon as a Mechanical Material" Proc. IEEE, 70 (1982) 420.

[5] K.S.J.Pister, M.W.Judy, S.R.Burgett and R.S.Fearing, "M icrofabricated Hinges ", Sensors \& Actuators (A), 33, (1992) pp. 249-256.

[6] C.-J. Kim, A.P.Pisano, R.S.Muller, "Silicon-Process Overhanging Microgripper", IEEE/ASME Jour. of Microelectromechanical Systems, 1 (1992) pp. 31-36.

[7] K.Ikuta and K. Hirowatari : Proc. IEEE Micro Electro Mechanical Systems, Fort Lauderdale, FL, 1993 (IEEE, Piscataway, 1993) p. 42. [8] W. S. N. Trimmer,"Micromechanical Systems" in Integrated Micro motion Systems (ed. F. Harashima), Elsevier Science Publ.,(1990) pp. $1-15$.

[9] L.-S. Fan, Y.-C. Tai, and R. S. Muller,"IC-processed electrostatic micromotors" Sensors \& Actuators 20 (1989) pp.41-48.

[10] M. Meheregany, P. Nagarkar, S. D. Senturia, and J. H. Lang, "Operation of Microfabricated Harmonic and Ordinary Side-Drive Motors" Proc. 3rd. IEEE MEMS Workshop, Napa Valley Feb.(1990) pp. 1-8.

[11] W. C. Tang, T.-C. H. Nguyen, and R. T. Howe,"Laterally driven polysilicon resonant microstructures" Sensors \& Actuators 20 (1989) pp. 25-32.

[12] F. C. M. Van De Pol, D. G. J. Wonnink M. Elwenspoek, and J. H. J. Fluitman"A Thermo-pneumatic Actuation Priciple for a Microminiature Pump and Other Micromechanical Devices" Sensors \& Actuators, 17 (1989) p.p. 139-143.

[13] M. Ataka, A. Omodaka, N. Takeshima and H. Fujita,"Polyimide Bimorph Actuators for a Ciliary Motion System", IEEE/ASME J. of Microelectromecanical Syst. vol.2, no.4(1993) pp.146-150.

[14] Y.-K. Kim, M. Katsurai, and H. Fujita,"A levitation-type linear synchronous microactuator using the Meissner effect of high-Tc superconductors",Sensors and Actuators A, 29(1991), pp. 143-150.

[15] S. Konishi and H. Fujita,"A Conveyance System Using Air Flow Based on the Concept od Distributed Micro Motion Systems" IEEE/ASME J. of Microelectromecanical Syst.,3 (1994 )54-58.

[16] T. Higuchi, M. Watanabe, K. Kudoh, "Precise Positioner Utilizing Rapid Deformations of a Piezo Electric Element", Joumal of the Japan Society of Precision Engineering, Vol. 54, No. 11, Nov. (1988).

[17] J. M. Younse, "Mirrors on a chip", IEEE Specturum, November (1993), pp. 27

[18] R. Sawada, H. Tanaka, O. Ohguchi, J. Shimada, and S. Hara, "Fabrication of Active Integrated Optical Micro-Encoder" Proc. 4th IEEE Workshop on Micro Electro Mechanical Systems, Nara, Japan, January 30-February 2 (1991) pp. 233-238.

[19] L. Y. Lin, S. S. Lee, K. S. J. Pister and M. C. Wu, "Threeidimentional micro-Fresnel optical elements fabricated by micromachining technique," Electron. Lett., Vol. 30, No. 5 (1994), pp. 448-449.

[20] S. Nakagawa, S. Shoji and M. Esashi, "Integrated Fluid Control Systems on a Silicon Wafer", in Micro System Technologies 90 (ed. H.Reichl), Springer-Verlag(1990), p.793.

[21] J. A. Walker, K. W. Goossen and S. C. Amey, "Fabrication of a Mechanical Anti-Reflection Switch for Fiber-to-the-Home Applications", IEEE/ASME J. of Microelectromechanical Syst. Vol. 5, No. 1, pp. 45-51 (1996).

[22] W. Tang, V. Temesvary, J. Jason Yao, Yu-Chong Tai, D. K. Miu, "Silicon Microactuators for Computer Disk Dives", Jpn. J. Appl. Phys., Vol. 35(1996), Part 1, No. 1B, January, pp. 350-356.

23] M. Washizu,"Electrostatic Manipulation of Biological Objects in Microfabricated Structures" in Integrated Micro motion Systems (ed F.
Harashima), Elsevier Science Publ., (1990) pp.417-432.

[24] D.Kobayashi, H.Fujita,"Design, Fabrication and Operation of a Micromachined Tunneling Control Unit", Trans. IEE of Japan, Vol. 116-E, No. 8(1996), pp. 339-344.

[25] T.W. Kenny, S.B. Waltman, J.K. Reynolds and W.J. Kaiser, "Micromachined silicon tunnel sensor for motion detection", Appl. Phys. Lett. 58 (1991) p. 100.

[26]Proc. of First Intn'l Conf. on Micro/Nano Technology for Space Application, Oct. 30-Nov. 3, 1995, League City, Texas, USA.

[27] H. Fujita, M. Ataka, S. Konishi, "Group work of distributed microactuators", Robotica(1996), Vol. 14, pp. 487-492.

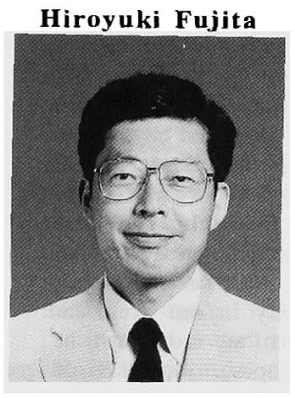

He received the B.S. degree in 1975 , the M.S. degree in 1977 and the Ph.D. degree in 1980 , all in electrical engineering, from the University of Tokyo, Tokyo, Japan. He is currently a Professor in the Institute of Indusrial Science at the University of Tokyo, where he joined the faculty in 1980. His current research interests are system design and fabrication technologies of microelectromecchanical systems. He also investigates autonomous distributed systems that mimic living organisms. 